\title{
The SCARB1 gene is associated with lipid response to dietary and pharmacological interventions
}

\author{
Yongjun Liu · Jose M. Ordovas · Guimin Gao $\cdot$ Michael Province · \\ Robert J. Straka $\cdot$ Michael Y. Tsai - Chao-Qiang Lai $\cdot$ Kui Zhang \\ Ingrid Borecki · James E. Hixson · David B. Allison · Donna K. Arnett
}

Received: 23 March 2008/Accepted: 6 May 2008/Published online: 10 June 2008

(C) The Japan Society of Human Genetics and Springer 2008

\begin{abstract}
The scavenger receptor class B type 1 (SCARB1) gene is a key component in the reverse cholesterol transport pathway and thus plays an important role in lipid metabolism. Studies suggest that the SCARB1 gene may contribute to variation in plasma lipid levels at fasting; however, the results have been inconsistent, and it is unclear whether SCARB1 may also influence lipid response to dietary and pharmacologic interventions. In this study, we examined genetic variation in the SCARBI gene in participants of the Genetics of Lipid Lowering Drugs and Diet Network (GOLDN) study for associations with basal lipid levels, changes in lipid measures after dietary fat intake, and fenofibrate treatment. We found that the exon 1
\end{abstract}

Electronic supplementary material The online version of this article (doi:10.1007/s10038-008-0302-2) contains supplementary material, which is available to authorized users.

\section{K. Arnett ( $\square)$}

Department of Epidemiology, School of Public Health,

University of Alabama at Birmingham,

220E Ryals Public Health Building,

1665 University Blvd., 1530 3rd Avenue South,

Birmingham, AL 35294-0022, USA

e-mail: arnett@uab.edu

Y. Liu · G. Gao · K. Zhang · D. B. Allison

Department of Biostatistics, School of Public Health,

University of Alabama at Birmingham,

Birmingham, AL, USA

\section{B. Allison}

Clinical Nutrition Research Center,

University of Alabama at Birmingham,

Birmingham, AL, USA

M. Province - I. Borecki

Division of Biostatistics, Washington University

School of Medicine, Saint Louis, MO, USA variant SCARB1_G2S was significantly associated with postfenofibrate change for triglycerides (TG) $(P=0.004)$. Subjects bearing SCARB1_G2S minor allele $A$ tend to have higher responsiveness to fenofibrate in lowering TG. In summary, our study suggested that the SCARBI gene may serve as a useful marker that predicts variation in baseline lipid levels, postprandial lipid response, and response to fenofibrate intervention.

Keywords Scavenger receptor class B type 1 . Lipid - Genetics · Fenofibrate · Postprandial

\section{Introduction}

Plasma triglyceride (TG) and cholesterol levels are major risk factors of coronary heart disease (CHD) (Cullen et al.

\author{
J. M. Ordovas · C.-Q. Lai \\ Department of Agriculture Human Nutrition Research \\ Center on Aging, Tufts University, Boston, MA, USA \\ M. Y. Tsai \\ Laboratory of Medicine and Pathology, \\ University of Minnesota, Minneapolis, MN, USA \\ R. J. Straka \\ Experimental and Clinical Pharmacology Department, \\ College of Pharmacy, University of Minnesota, \\ Minneapolis, MN, USA \\ J. E. Hixson \\ Human Genetics Center, \\ University of Texas Health Science Center, \\ Houston, TX, USA
}


1997). High-density lipoprotein cholesterol (HDL-C) levels inversely correlated with CHD risk, suggesting HDL-C protects against atherosclerosis (Gordon and Rifkind 1989; Uiterwaal et al. 1994). TGs, low-density lipoprotein (LDL) and HDL particles and particle sizes are highly reproducible and are sensitive to dietary manipulation and lipidlowering drugs (Tsai et al. 1992; Yuan et al. 1994). Studies showed that both fasting lipid levels and postprandial lipid response are modulated by genetic factors (Mero et al. 1998; Ordovas 2001). In addition, several lines of evidence suggest that lipid response to pharmacological interventions is highly variable and has genetic control (Suter et al. 2001; Durstine et al. 2001; Kuller et al. 2001). However, these underlying genetic factors are largely unknown.

The scavenger receptor class $\mathrm{B}$ type 1 (SCARB1) gene is the first reported HDL receptor (Acton et al. 1996). $S C A R B 1$ mediates selective uptake of HDL-C without degradation of entire HDL particles (Acton et al. 1996). Overexpression of $S C A R B 1$ in mice resulted in dramatic reductions in plasma HDL-C concentrations (Kozarsky et al. 1997). Conversely, suppression of SCARB1 expression was related to a marked increase in plasma HDL-C, characterized by enlarged cholesterol-rich HDL particles and impaired HDL-C clearance (Rigotti et al. 1997). As a multilipoprotein receptor, $S C A R B 1$ also regulates the concentrations of LDL-C and very-low-density lipoprotein (VLDL) (Acton et al. 1996; Ueda et al. 1999). Interestingly, $S C A R B 1$ is likely to be involved in intestinal absorption of TGs (Hauser et al. 1998). Importantly, the $S C A R B 1$ gene is located on chromosome $12 \mathrm{q} 24$, a region showing significant linkage to plasma HDL-C and TG levels (Feitosa et al. 2005). Several previous studies also reported associations between the SCARB1 gene polymorphisms and plasma cholesterol levels in humans; however, the results have been inconsistent (Acton et al. 1999) and it is unclear whether the SCARBl gene is involved in modulating postprandial lipid response and response to pharmacological interventions.

Stimulated by the importance of $S C A R B 1$ in lipid metabolism, we systemically investigated whether the $S C A R B 1$ gene contributes to baseline lipid levels, lipid response to dietary fat challenge, and, for the first time, TG-lowering drug therapy in participants of the Genetics of Lipid Lowering Drugs and Diet Network (GOLDN) study.

\section{Materials and methods}

Study population

Our study population came from the GOLDN study (http://www.biostat.wustl.edu/goldn/). The participants of
GOLDN study were mainly rerecruited from two National Heart, Lung, and Blood Institute Family Heart Study (FHS) field centers: Minneapolis, MN, and Salt Lake City, UT. Nearly all subjects were of European ancestry. Eligibility criteria were: (1) $\geq 18$ years of age, (2) fasting TGs $<1,500 \mathrm{mg} / \mathrm{dl}$, (3) willing to participate in the study and attend the scheduled clinic exams, (4) member of a family with at least two members in a sibship, (5) aspartate aminotransferase (AST) and alanine aminotransferase (ALT) results within normal range, and (6) creatinine $\leq 2.0 \mathrm{mg} / \mathrm{dl}$. Exclusion criteria were: (1) history of liver, kidney, pancreas, or gall bladder disease or malabsorption, (2) current pregnancy, (3) insulin use, (4) use of lipid-lowering drugs (including prescription, over the counter, and nutriceuticals; volunteers taking these agents were withdrawn from them at least 4 weeks prior to the study with physician's approval) (5) use of warfarin, (6) women of childbearing potential not using an acceptable form of contraception, (7) known hypersensitivity to fenofibrate, and (8) history of pancreatitis within 12 months prior to enrollment.

Our previous studies demonstrated that Caucasians in Utah and Minnesota were homogeneous and thus pooling data across centers would not threaten the validity of this study (Pankow et al. 2002).

\section{Dietary and pharmacological interventions}

All family members underwent a baseline screening visit (visit 0). Eligible subjects were asked to participate in a clinical examination with dietary fat intake and the fenofibrate trial. The dietary fat challenge was conducted to assess baseline and postprandial TGs and related phenotypes. The fat challenge consisted of eating a test meal during a 15-min period and returning to the clinic 3.5 and $6 \mathrm{~h}$ later to collect blood samples. The caloric intake of the intervention meal was determined by body surface area. The meal derived $83 \%$ of calories from fat and contained 700 calories per meter squared of body surface area. We measured total TGs, TG in chylomicrons, VLDL and TG remnant particles, HDL and LDL particle sizes, total cholesterol, LDL-C, and HDL-C at baseline and at 3.5 and $6 \mathrm{~h}$ after consumption of the meal.

This fat challenge was done twice, once while participants were taking no lipid-lowering medications and once while taking fenofibrate. The fenofibrate intervention consisted of a 3-week treatment period in which participants took oral fenofibrate $160 \mathrm{mg}$ tablets once daily. We measured lipids twice after a minimum fast of $10 \mathrm{~h}$ on the last 2 days of the treatment period (visits 3 and 4). The intervention procedure is displayed in supplementary data (Supplementary Fig. 1, ESM). 
Plasma lipid and lipoprotein measurements

Fasting, 3.5, and $6 \mathrm{~h}$ postprandial lipoprotein samples were centrifuged within $20 \mathrm{~min}$ of collection at 2,000 $\mathrm{g}$ for $15 \mathrm{~min}$ at $4^{\circ} \mathrm{C}$. Plasma samples of each participant were stored at $4^{\circ} \mathrm{C}$ until completion of the treatment period. All samples from a single participant were analyzed within the same batch. TGs were measured using a glycerol blanked enzymatic method (Trig/GB, Roche Diagnostics Corporation, Indianapolis, IN, USA) on the Roche/Hitachi 911 Automatic Analyzer (Roche). Cholesterol was measured on the Hitachi 911 using a cholesterol esterase-cholesterol oxidase reaction (Chol R1, Roche). The same reaction was also used to measure HDL-C after precipitation of nonHDL-C with magnesium/dextran. LDL-C was measured by a homogeneous direct method (LDL Direct Liquid Select $^{\mathrm{TM}}$ Cholesterol Reagent, Equal Diagnostics, Exton, PA, USA) on the Hitachi 911. TGs were measured at all time points, i.e., $0,3.5$, and $6 \mathrm{~h}$ before and after fenofibrate trial. HDL-C and LDL-C were only measured at baseline before and after fenofibrate exposure.

Plasma lipoprotein particles and subclass distributions were determined by proton nuclear magnetic resonance (NMR) spectroscopy as previously described (Ordovas et al. 2000; Otvos et al. 1992). Each profile displayed the concentrations of three VLDL, one IDL, three LDL, and three HDL subclasses and the weighted-average particle sizes of VLDL, LDL, and HDL. The lipoprotein subclass categories used were large LDL (21.3-27.0 nm), intermediate LDL (19.8-21.2), small LDL (18.3-19.7 nm), large HDL (8.8-13.0 nm), intermediate HDL (7.8-8.8 nm), and small HDL (7.3-7.7 nm). Levels of LDL and HDL subclasses are expressed in nanomole units of cholesterol. LDL and HDL subclass distributions determined by gradient gel electrophoresis and NMR have been shown to be closely correlated (Otvos et al. 1992). HDL, LDL, and VLDL particles and subclass were measured at all time points $(0,3.5$, and $6 \mathrm{~h})$ before and after fenofibrate trial.

\section{SNP selection and genotyping}

Single nucleotide polymorphisms (SNPs) were identified by searching public databases such as the Single Nucleotide Polymorphism Database (dbSNP) (http://www.ncbi. nlm.nih.gov/SNP/). We selected seven SNPs at the $S C A R B 1$ gene locus based on the following criteria in order of importance in our selection scheme: (1) validation status, (2) functional relevance and importance, (3) degree of heterozygosity, i.e., minor allele frequencies (MAF) $>0.0$, and (4) previous evidence of association with lipid measurements.

SNPs were genotyped using the $5^{\prime}$ nuclease allelic discrimination Taqman assay with allelic-specific probes on the ABI Prism 7900HT Sequence Detection System (Applied Biosystems, Foster City, CA, USA) according to standard laboratory protocols. We used GRR software (Abecasis et al. 2001) to detect pedigree errors by graphically inspecting the distribution for marker allele sharing among pairs of family members or all pairs of individuals. The SNP allele frequencies were estimated via a maximum-likelihood method (Boehnke 1991). For each SNP, a $\chi^{2}$ test was used to examine deviation of SNP genotypes from Hardy-Weinberg equilibrium (HWE) (Fisher 1934). The overall genotyping error and missing rate was $\sim 1 \%$.

Statistical analyses

Pairwise linkage disequilibrium (LD) between SNPs was calculated using the normalized measure, Lewontin's $D$, (Lewontin 1964), using the publicly available Haploview version 3.3 software (Barrett et al. 2005). Normality for continuous variables was checked. After logarithmic transformations for TGs, HDL-C, and HDL particle size, they approximately follow normal distribution.

Associations were tested between the SCARB1 SNPs and a number of lipid profiles, which included: (1) baseline lipid levels (including TGs, HDL-C, LDL-C, HDL, and LDL particles and particle sizes), (2) changes of lipid profile between pre- and postfenofibrate treatments, (3) changes of lipid profile at $0-3.5 \mathrm{~h}$ (which, biologically, represents the absorption phase of TGs after a fat meal), and (4) changes of lipid profile at 3.5-6 h (which, biologically, represents the clearance phase of TGs after a fat meal).

Genotype-phenotype association analyses were performed using a linear mixed model implemented in SAS (version 9.1, SAS Institute, Cary, NC, USA). In the model, genotypes were treated as fixed effects, and the dependencies among members within each family were treated as random effects. Age, age $^{2}$, age $^{3}$, gender, and field center were included as covariates. The model can be defined as:

$y_{i t}=\alpha+\beta \times t+\gamma_{i \alpha}+\gamma_{i \beta} \times t+\varepsilon_{i t}$

where $y_{i t}$ is the phenotype of individual $i$ measured at time point $t ; \alpha$ and $\beta$ are the intercept and slope for the population level effects (fixed effects), respectively, and $\gamma_{i \alpha}$ and $\gamma_{i \beta}$ are for the individual level effects (random effects), respectively, and $\varepsilon_{i t}$ is the residual. The model was implemented in the PROC MIXED procedure in SAS. As the growth curve slope $\left(\beta+\gamma_{i \beta}\right)$ eliminates the noise $\varepsilon_{i t}$, it represents an accurate and stable estimate of lipid changes.

Haplotypes were reconstructed based on the seven SNPs. The software MERLIN version 1.0.1 (Abecasis et al. 2002) was used for haplotype inference. MERLIN can accommodate LD between SNPs in haplotype inference 
(Abecasis and Wigginton 2005). Association analysis for haplotypes was conducted using a mixed model similar to that for single SNPs. In addition, a global test was performed to examine whether phenotype differences existed among all haplotypes simultaneously.

\section{Results}

Study population

Characteristics of the study subjects are shown in Table 1. A total of 1,327 subjects (639 men and 688 women) from
148 families were genotyped. Of these 1,327 subjects, 861 subjects (427 men and 434 women) went through the fenofibrate trial and had complete lipid phenotype (TGs, HDL-C, and LDL-C) and genotype data. Men ranged in age from 18.0 to $87.6(50.6 \pm 15.9)$ years, and women ranged from 18.0 to $87.2(51.1 \pm 15.8)$ years. For both men and women at all the time points, TG, LDL-C, and LDL particles after fenofibrate treatment were significantly lower than those before fenofibrate treatment $(P<0.001)$. Conversely, HDL-C and HDL particles after fenofibrate treatment were significantly higher than those before fenofibrate treatment $(P<0.001)$. Postfenofibrate HDL sizes generally increased compared with those before fenofibrate

Table 1 Characteristics of study subjects

\begin{tabular}{|c|c|c|c|c|c|c|}
\hline \multirow[t]{2}{*}{ Measure } & \multicolumn{3}{|l|}{ Prefenofibrate } & \multicolumn{3}{|l|}{ Postfenofibrate } \\
\hline & Baseline & $3.5 \mathrm{~h}$ & $6 \mathrm{~h}$ & Baseline & $3.5 \mathrm{~h}$ & $6 \mathrm{~h}$ \\
\hline \multicolumn{7}{|l|}{ Men } \\
\hline Age (year) & $50.6(15.9)$ & - & - & - & - & - \\
\hline Triglyceride (mg/dl) & $153.41(142.01)$ & 286.05 (193.49) & $270.41(219.68)$ & $99.18(59.83)$ & $190.91(109.88)$ & $164.41(107.41)$ \\
\hline HDL-C (mg/dl) & $41.57(9.84)$ & NA & NA & 43.67 (9.99) & NA & NA \\
\hline LDL-C (mg/dl) & $123.17(30.32)$ & NA & NA & $111.45(32.79)$ & NA & NA \\
\hline HDL particles $(\mathrm{nmol} / \mathrm{L})$ & $29.13(4.98)$ & $28.64(4.83)$ & $29.71(4.61)$ & $30.57(5.36)$ & $30.47(4.86)$ & 31.44 (4.99) \\
\hline Large & $4.71(2.70)$ & $4.24(2.91)$ & $4.39(3.06)$ & $4.42(2.31)$ & $4.24(2.55)$ & $4.38(2.71)$ \\
\hline Intermediate & $1.99(2.67)$ & $4.01(3.58)$ & $5.80(4.39)$ & $4.15(3.90)$ & $6.65(4.53)$ & $8.78(5.09)$ \\
\hline Small & $22.42(5.02)$ & $20.39(5.08)$ & $19.50(5.39)$ & $22.00(6.06)$ & $19.56(5.97)$ & $18.27(6.52)$ \\
\hline LDL particles $(\mathrm{nmol} / \mathrm{L})$ & $1445.10(461.59)$ & 1420.64 (454.14) & $1429.91(464.17)$ & 1275.65 (397.52) & $1267.66(403.91)$ & $1267.28(414.65)$ \\
\hline Large & $319.12(228.59)$ & $357.44(242.54)$ & 380.15 (253.89) & $383.93(205.20)$ & $402.42(211.15)$ & $433.01(213.67)$ \\
\hline Intermediate & $48.17(52.05)$ & $79.76(74.32)$ & $73.59(69.87)$ & $35.01(40.01)$ & $47.36(46.97)$ & $43.42(39.25)$ \\
\hline Small & $1077.80(520.89)$ & 983.43 (502.99) & 976.16 (533.93) & $856.71(394.12)$ & 817.87 (403.30) & $790.84(423.83)$ \\
\hline HDL size (nm) & $8.65(0.38)$ & $8.66(0.41)$ & $8.73(0.40)$ & $8.85(0.40)$ & $8.95(0.41)$ & $9.02(0.39)$ \\
\hline LDL size $(\mathrm{nm})$ & $20.48(0.78)$ & $20.61(0.78)$ & $20.68(0.80)$ & $21.01(0.57)$ & $21.05(0.60)$ & $21.12(0.60)$ \\
\hline VLDL size (nm) & $51.38(7.42)$ & $55.72(8.37)$ & $58.86(9.88)$ & $51.56(8.89)$ & $55.63(9.55)$ & $61.09(13.25)$ \\
\hline \multicolumn{7}{|l|}{ Women } \\
\hline Age (year) & $51.1(15.8)$ & - & - & - & - & - \\
\hline Triglyceride (mg/dl) & $125.19(82.22)$ & $225.86(137.21)$ & $204.32(163.28)$ & $80.67(47.30)$ & $150.42(89.80$ & $128.52(88.09)$ \\
\hline HDL-C (mg/dl) & $52.29(13.68)$ & NA & NA & $55.03(14.23)$ & NA & NA \\
\hline LDL-C (mg/dl) & $119.63(32.15)$ & NA & NA & $97.19(28.83)$ & NA & NA \\
\hline HDL total (nmol/L) & $32.69(5.68)$ & $31.94(5.73)$ & $32.25(5.54)$ & $34.24(5.97)$ & $33.51(5.77)$ & 33.77 (5.76) \\
\hline Large & $7.80(3.58)$ & $7.60(3.83)$ & 7.73 (3.99) & $7.00(3.51)$ & $7.66(4.15)$ & $8.05(4.52)$ \\
\hline Intermediate & $3.92(4.09)$ & $6.44(4.53)$ & $9.34(5.27)$ & $7.63(4.97)$ & $10.29(5.02)$ & $12.98(5.42)$ \\
\hline Small & $20.96(5.92)$ & $17.89(6.15)$ & $15.17(6.92)$ & $19.60(7.09)$ & $15.55(7.03)$ & $12.73(7.29)$ \\
\hline LDL total $(\mathrm{nmol} / \mathrm{L})$ & $1313.24(476.05)$ & $1285.90(474.76)$ & $1299.91(483.46)$ & 1147.46 (353.64) & 1129.59 (341.16) & $1147.83(338.74)$ \\
\hline Large & 488.69 (284.94) & $530.79(530.79)$ & $577.02(287.76)$ & 410.10 (197.94) & 442.51 (210.97) & 477.38 (226.65) \\
\hline Intermediate & $37.62(52.68)$ & $56.71(61.01)$ & $54.30(56.37)$ & $30.44(37.87)$ & $40.23(38.18)$ & 42.03 (39.09) \\
\hline Small & $786.91(552.84)$ & 698.39 (537.35) & $668.58(553.83)$ & 706.92 (337.14) & $646.84(325.63)$ & $628.41(328.63)$ \\
\hline HDL size (nm) & $8.85(0.40)$ & $8.95(0.41)$ & $9.02(0.39)$ & $9.025(0.43)$ & $9.067(0.46)$ & $9.14(0.45)$ \\
\hline LDL size $(\mathrm{nm})$ & $21.09(0.86)$ & $21.22(0.85)$ & $21.33(0.83)$ & $21.00(0.57)$ & $21.05(0.60)$ & $21.12(0.60)$ \\
\hline VLDL size $(\mathrm{nm})$ & $51.38(8.18)$ & 55.77 (8.59) & $60.50(10.81)$ & $51.56(8.89)$ & $55.63(9.55)$ & $61.09(13.25)$ \\
\hline
\end{tabular}

Values are reported as mean (standard deviation) or as number

$H D L-C$ high-density lipoprotein cholesterol, $L D L-C$ low-density lipoprotein cholesterol, $V L D L$ very-low-density lipoprotein, $N A$ not applicable 
treatment at each corresponding time point for both men and women. The changes of VLDL and LDL particle sizes before and after treatment were not in a consistent pattern for either men or women.

\section{SNP genotyping and linkage disequilibrium}

Information on the seven tested SNPs is summarized in Supplementary Table 1 (ESM). All SNPs were in HardyWeinberg equilibrium. Allele frequencies of all SNPs were $>0.10$. LD coefficients (Lewontin's $D^{\prime}$ ) between each pair of SNPs are shown in Supplementary Table 2 (ESM). We found substantial $\mathrm{LD}\left(D^{\prime} \geq 0.50\right)$ among several pairs of SNPs. The strongest $\mathrm{LD}\left(D^{\prime}=0.904\right)$ was found between SCARB1_I51973 (intron 5) and SCARB1_A350A (exon 8).

\section{Association analyses for single SNPs}

Table 2 summarizes the association results between SNPs and lipid response to fenofibrate treatment in the total sample. Overall, fenofibrate was associated with a $27.2 \mathrm{mg} / \mathrm{dl}$ reduction in TG level. The SNP SCARB1_G2S was associated with fenofibrate response for both TG $(P=0.004)$ and HDL-C $(P=0.01)$. The SNP SCARB1_I82699 was associated with fenofibrate response for TG $(P=0.02)$. Figure 1 displays the changes of HDL-C and TG for SCARB1_G2S genotypes. It can be seen that subjects bearing allele $A$ had larger changes for both HDL-C and TG, suggesting higher response to fenofibrate treatment by increasing plasma HDL$\mathrm{C}$ level and lowering TG level.

Table 3 summarizes association results of lipid measures at baseline $(0 \mathrm{~h})$ and lipid changes across time points of $0-3.5 \mathrm{~h}(0 / 3.5)$ and $3.5-6 \mathrm{~h}(3.5 / 6)$. The most pronounced association was found for SNP SCARB1_G2S. In the prefenofibrate phase, a significant association was

Table 2 Association of SCARB1 single nucleotide polymorphisms (SNPs) with lipid response to fenofibrate treatment

\begin{tabular}{llll}
\hline & $P$ value* & \\
\cline { 2 - 4 } & HDL-C change & LDL-C change & TG change \\
\hline SCARB1_G2S & $\mathbf{0 . 0 1}$ & 0.65 & $\mathbf{0 . 0 0 4}$ \\
SCARB1_I9107 & 0.22 & 0.23 & 0.12 \\
SCARB1_19960 & 0.07 & 0.38 & 0.78 \\
SCARB1_I30026 & 0.65 & 0.42 & 0.35 \\
SCARB1_I51973 & 0.56 & 0.89 & 0.85 \\
SCARB1_A350A & 0.77 & 0.86 & 0.09 \\
SCARB1_I82699 & 0.39 & 0.91 & $\mathbf{0 . 0 2}$ \\
\hline
\end{tabular}

*Numbers given in the table are $P$ values of the association analyses using linear mixed models. Associations with SNPs were tested for changes of HDL-C, LDL-C, and TG before and after fenofibrate treatments. $P$ values of $\leq 0.05$ are in bold found between SCARB1_G2S and baseline HDL $(P=0.001), \mathrm{HDL}_{0-3.5}(P=0.04)$, and changes of HDL particle size at time period $0-3.5 \mathrm{~h} \quad(P=0.03)$. $S C A R B 1 \_$G2S also showed associations with baseline TG $(P=0.004), \quad \mathrm{TG}_{0-3.5} \quad(P=0.005)$, and baseline $\mathrm{LDL}$ $(P=0.03)$ (Table 3).

For postfenofibrate lipid measures, significant associations were observed for SCARB1_G2S with baseline HDL particles $(P=0.002), \mathrm{HDL}_{3.5-6}$, and HDL size at time period 3.5-6 h $(P=0.0002)$. However, no association was found between SCARB1_G2S and baseline TG or TG response to fat loading.

\section{Association analyses for haplotypes}

A total of 51 haplotypes was observed in our family data. To maximize the statistical power and minimize potential bias caused by the small number of subjects bearing a specific haplotype, we only tested associations between common haplotypes (allele frequencies $\geq 0.05$ ) and lipid measures. Table 4 illustrates the frequencies and association information of the six common haplotypes $(H 1-H 6)$. No significant association was observed between any single haplotype with response to fenofibrate treatment. However, haplotype $H 1$ showed significant association with prefenofibrate baseline HDL $(P=0.002)$ and marginal association with $\mathrm{HDL}_{3.5-6}(P=0.07)$. $H 1$ was also association with prefenofibrate $\mathrm{TG}_{0-3.5}(P=0.02)$. Haplotype $H 2$ was associated with postfenofibrate $\mathrm{HDL}_{3.5-6}$ and $\mathrm{HDL}$

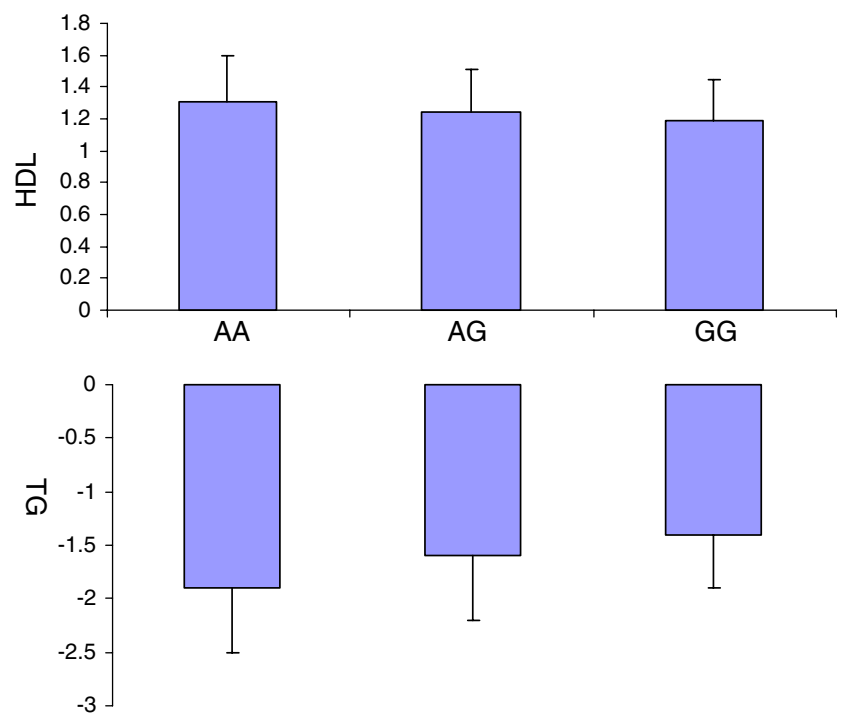

Fig. 1 Changes in high-density lipoprotein cholesterol (HDL-C) and triglycerides (TG) with SCARB1_G2S genotypes in response to fenofibrate treatment. Changes (mean \pm standard error) of HDL-C and TG ( $Y$ axis) were growth-curve slopes estimated from the mixed models 
Table 3 Association tests of SCARB1 single nucleotide polymorphisms (SNPs) with baseline lipid measures and response to dietary fat

\begin{tabular}{|c|c|c|c|c|c|c|c|c|c|c|c|c|c|c|c|c|c|c|}
\hline \multirow[t]{2}{*}{ SNP } & \multicolumn{3}{|l|}{ HDL } & \multicolumn{3}{|l|}{ LDL } & \multicolumn{3}{|c|}{ HDL size } & \multicolumn{3}{|c|}{ LDL size } & \multicolumn{3}{|c|}{ VLDL size } & \multicolumn{3}{|l|}{ TGs } \\
\hline & 0 & $0 / 3.5$ & $3.5 / 6$ & 0 & $0 / 3.5$ & $3.5 / 6$ & 0 & $0 / 3.5$ & $3.5 / 6$ & 0 & $0 / 3.5$ & $3.5 / 6$ & 0 & $0 / 3.5$ & $3.5 / 6$ & 0 & $0 / 3.5$ & $3.5 / 6$ \\
\hline \multicolumn{19}{|l|}{ Pre-fenofibrate } \\
\hline SCARB1_G2S & 0.001 & 0.04 & 0.05 & 0.03 & 0.13 & 0.82 & 0.83 & 0.05 & 0.41 & 0.54 & 0.25 & 0.41 & 0.12 & 0.31 & 0.50 & 0.004 & 0.005 & 0.31 \\
\hline SCARB1_I9107 & 0.85 & 0.44 & 0.66 & 0.67 & 0.51 & 0.52 & 0.02 & 0.70 & 0.97 & 0.36 & 0.52 & 0.97 & 0.22 & 0.53 & 0.98 & 0.77 & 0.70 & 0.65 \\
\hline SCARB1_19960 & 0.54 & 0.46 & 0.41 & 0.39 & 0.24 & 0.52 & 0.05 & 0.85 & 0.99 & 0.77 & 0.90 & 0.99 & 0.71 & 0.44 & 0.56 & 0.94 & 0.15 & 0.32 \\
\hline SCARB1_I30026 & 0.38 & 0.80 & 0.15 & 0.76 & 0.37 & 0.67 & 0.81 & 0.85 & 0.73 & 0.76 & 0.17 & 0.73 & 0.08 & 0.73 & 0.44 & 0.47 & 0.44 & 0.19 \\
\hline SCARB1_I51973 & 0.65 & 0.92 & 0.73 & 0.90 & 0.71 & 0.95 & 0.55 & 0.65 & 0.21 & 0.81 & 0.86 & 0.21 & 0.57 & 0.26 & 0.58 & 0.43 & 0.21 & 0.31 \\
\hline SCARB1_A350A & 0.20 & 0.13 & 0.53 & 0.10 & 0.68 & 0.02 & 0.40 & 0.67 & 0.41 & 0.42 & 0.21 & 0.41 & 0.14 & 0.87 & 0.71 & 0.28 & 0.36 & 0.48 \\
\hline SCARB1_I82699 & 0.92 & 0.78 & 0.90 & 0.29 & 0.20 & 0.58 & 0.10 & 0.23 & 0.72 & 0.09 & 0.14 & 0.72 & 0.87 & 0.95 & 0.48 & 0.04 & 0.37 & 0.01 \\
\hline \multicolumn{19}{|l|}{ Postfenofibrate } \\
\hline SCARB1_G2S & 0.002 & 0.37 & 0.01 & 0.36 & 0.49 & 0.70 & 0.76 & 0.04 & 0.0002 & 0.27 & 0.29 & 0.19 & 0.29 & 0.42 & 0.57 & 0.59 & 0.29 & 0.69 \\
\hline SCARB1_I9107 & 0.62 & 0.26 & 0.47 & 0.21 & 0.60 & 0.26 & 0.14 & 0.99 & 0.98 & 0.35 & 0.28 & 0.67 & 0.63 & 0.55 & 0.70 & 0.16 & 0.88 & 0.57 \\
\hline SCARB1_19960 & 0.14 & 0.46 & 0.60 & 0.004 & 0.10 & 0.82 & 0.03 & 0.54 & 0.46 & 0.63 & 0.21 & 0.19 & 0.04 & 0.56 & 0.59 & 0.36 & 0.54 & 0.24 \\
\hline SCARB1_I30026 & 0.84 & 0.65 & 0.58 & 0.53 & 0.74 & 0.54 & 0.87 & 0.85 & 0.85 & 0.78 & 0.85 & 0.91 & 0.93 & 0.45 & 0.76 & 0.80 & 0.79 & 0.50 \\
\hline SCARB1_I51973 & 0.78 & 0.64 & 0.44 & 0.97 & 0.72 & 0.42 & 0.49 & 0.34 & 0.46 & 0.11 & 0.15 & 0.08 & 0.15 & 0.08 & 0.82 & 0.22 & 0.89 & 0.99 \\
\hline SCARB1_A350A & 0.81 & 0.28 & 0.33 & 0.14 & 0.11 & 0.26 & 0.49 & 0.24 & 0.78 & 0.80 & 0.51 & 0.48 & 0.44 & 0.24 & 0.72 & 0.30 & 0.11 & 0.15 \\
\hline SCARB1_I82699 & 0.39 & 0.31 & 0.03 & 0.54 & 0.13 & 0.02 & 0.54 & 0.27 & 0.36 & 0.14 & 0.11 & 0.16 & 0.77 & 0.25 & 0.01 & 0.36 & 0.41 & 0.31 \\
\hline
\end{tabular}

$S N P$ single nucleotide polymorphism, $H D L$ high-density lipoprotein, $L D L$ low-density lipoprotein, $V L D L$ very-low-density lipoprotein

$P$ values of association tests using linear mixed models. Associations were tested for lipid measures at baseline, and changes of lipid measures over time $0-3.5(0 / 3.5) \mathrm{h}$ and 3.5-6 (3.5/6) h for pre-fenofibrate and postfenofibrate, separately. $P$ values of $\leq 0.05$ are in bold

size $_{3.5-6}$. Other haplotypes not specified here showed no significantly association with any lipid phenotypes.

\section{Discussion}

In this study, we systemically investigated the relationship between the SCARB 1 gene and lipid profiles at the baseline and postprandial states, as well as lipid lowering treatment. Dietary fat challenges and pharmacologic intervention are two potent stimuli that produce large but highly variable changes in lipid profile (Suter et al. 2001; Durstine et al. 2001; Kuller et al. 2001). We undertook two interventions (i.e., dietary fat challenge and pharmacologic intervention), because by studying contexts that affect both axes (i.e., increasing and decreasing TGs) we may gain novel insight into underlying mechanisms by which the SCARB1 gene may regulate lipids. The most interesting finding was with the SNP SCARB1_G2S. Allele A of this SNP, compared with the alternative allele $G$, was associated with a higher response to fenofibrate by lowering TG levels and increasing plasma HDL-C levels (but the significance with HDL-C disappeared after adjusting for multiple testing).

Several previous studies examined association between the SCARB1 gene polymorphisms and lipid measures in different human populations. In a white population, male carriers of the two allele (1/2) of SCARB1_G2S had increased HDL-C and reduced LDL-C and TGs concentrations at baseline (Acton et al. 1999). In a dietary intervention study, carriers of this allele appeared to be more responsive to changes in dietary saturated fat intake, as they exhibited a greater increase in LDL-C compared with 1/1 individuals (Pérez-Martínez et al. 2003). In the Framingham Study, diabetic subjects carrying the $S C A R B 1$ G2S $A$ allele had significantly lower LDL-C and HDL-C concentrations (Osgood et al. 2003). Our results reported here are in agreement with these earlier findings. An important extension of our study is that we, for the first time, found the SCARB1 gene is also related to lipid response to pharmacological (fenofibrate) treatment.

Mice studies showed that the genetic expression of SCARB1 modifies the metabolism of HDL-C. Overexpression of $S C A R B 1$ in the liver lowers plasma values for HDL-C and increases the concentration and biliary secretion of cholesterol (Kozarsky et al. 1997; Wang et al. 1998). In contrast, the total suppression of its expression increases plasma HDL-C and lowers cholesterol content in bile and the suprarenal gland. After feeding them a meal containing $\left[{ }^{14} \mathrm{C}\right]$ cholesterol and $\left[{ }^{3} \mathrm{H}\right]$ triolein, mice with $S C A R B 1$ overexpression primarily in the intestine presented a rise in intestinal absorption of both lipids, which was not due to a defect in chylomicron clearance nor to a change in bile flow or bile acid content (Bietrix et al. 2006), suggesting that SCARBI acts as a multiligand 


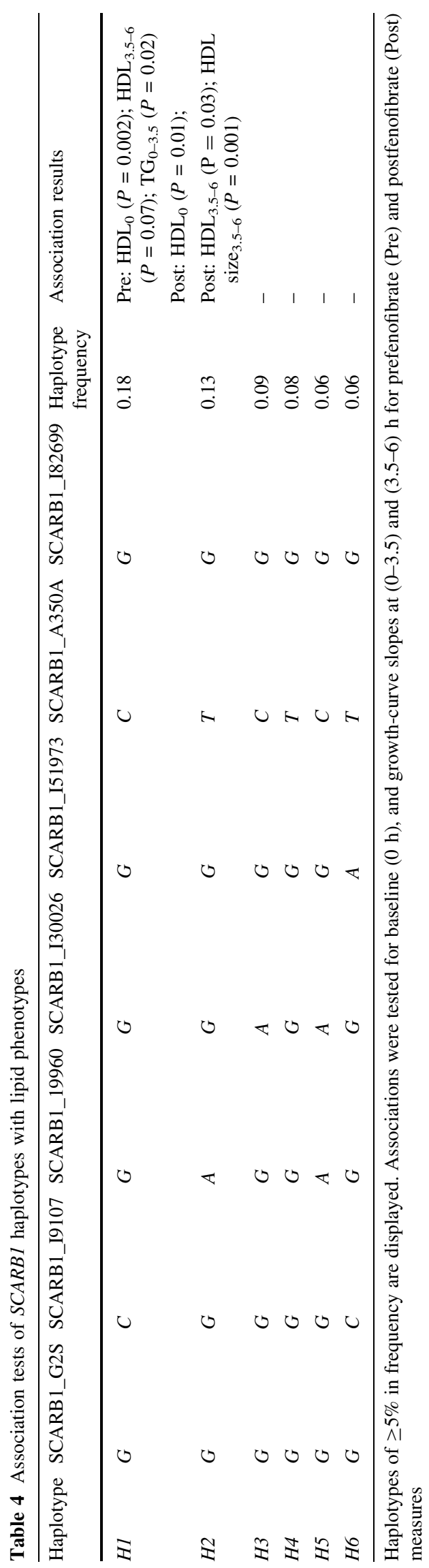

transporter in the small intestine and is responsible for the accelerated uptake of cholesterol and TG hydrolysis products. The mechanism by which SCARB1 delivers cholesterol esters to cells is not well understood, but it appears to be different from the well-characterized LDL receptor endocytic pathway (Brown and Goldstein 1986). It is likely that $S C A R B 1$ mediates uptake of lipids from both LDL and HDL, and this uptake is associated with an increase in cholesterol esterification (Stangl et al. 1998).

The physiological mechanism of SCARB1 in the postprandial lipemic response and pharmacologic intervention awaits further molecular/physiological studies for elucidation. Nonetheless, our findings from this study, along with those of others, suggest a potential mechanism by which $S C A R B 1$ gene polymorphisms may be related to a modified hepatic and/or intestinal expression of the SCARB1 gene, through which SCARBI influences lipid absorption and clearance. In particular, the exon 1 polymorphism of $S C A R B 1 \_G 2 S$ results in an amino acid change in the protein, which may modify receptor activity. Based on the current understanding of this exon 1 variant, it is unlikely that it directly impairs selective cholesterol uptake. This is because selective cholesterol uptake resides primarily in the extracellular domain of the receptor, but the exon 1 amino acid change occurs in its intracellular N-terminus. Further in vitro and in vivo studies are needed to elucidate the exact functional effects of this $S C A R B 1$ exon 1 variant.

A major strength of this study is that participants underwent rigorous interventions of both fat-loading dietary challenge and fenofibrate trial, which provided a valuable resource for investigating the SCARBI gene regarding its relationship with baseline lipid levels, postprandial lipid response, and response to pharmacological treatment. Despite this asset, our study may have some limitations. First, although multiple SNPs were investigated across the gene, marker density was not sufficient to cover the whole gene. To examine the gene more comprehensively, further studies by testing more SNPs, especially tag SNPs that cover all LDs in SCARB1, should be pursued. Second, a number of tests were performed due to the multiple lipid measures and SNPs involved. After correction for multiple testing, some identified associations only reached nominal significance or even disappeared. For example, the association between SCARB1_G2S and HDL$\mathrm{C}$ in response to fenofibrate treatment turned out to be nonsignificant after adjusting for multiple testing. However, as the tested lipid measures were highly correlated and the tested SNPs were in LD, Bonferroni correction may be too conservative. Third, as our analyses were exploratory, the results await replication studies and eventually functional studies to validate them. Due to the associated high costs and difficulties in compliance with the strict dietary and pharmacological interventions, we currently 
lack powerful independent data sets (including those from other groups) to replicate our study findings. We will pursue replication/confirmation in future endeavors.

In summary, this study suggests that SCARBI gene polymorphisms may serve as useful markers to predict baseline lipid measures, postprandial lipid response, and fenofibrate response. Our results may have significant clinical implications if confirmed by future studies.

Acknowledgments We thank the families for their participation in this research. This work was supported by the National Institute of Health Heart, Lung, and Blood Institute grant U 01 HL72524, genetic and environmental determinants of triglycerides. We acknowledge Abbott Laboratories (Abbott Park, IL, USA) for their supply of study medication for this project. Dr. David B. Allison is supported by NIH grant 3P30DK056336. Dr. Kui Zhang is supported by NIH grant R01GM74913.

Conflict of interest We declare that we have no duality or conflict of interest.

\section{References}

Abecasis GR, Wigginton JE (2005) Handling marker-marker linkage disequilibrium: pedigree analysis with clustered markers. Am J Hum Genet 77(5):754-767

Abecasis GR, Cherny SS, Cookson WO, Cardon LR (2001) GRR: graphical representation of relationship errors. Bioinformatics 17(8):742-743

Abecasis GR, Cherny SS, Cookson WO, Cardon LR (2002) Merlinrapid analysis of dense genetic maps using sparse gene flow trees. Nat Genet 30(1):97-101

Acton S, Rigotti A, Landschulz KT, Xu S, Hobbs HH, Krieger M (1996) Identification of scavenger receptor SR-BI as a high density lipoprotein receptor. Science 271(5248):518-520

Acton S, Osgood D, Donoghue M, Corella D, Pocovi M, Cenarro A, Mozas P, Keilty J, Squazzo S, Woolf EA, Ordovas JM (1999) Association of polymorphisms at the SR-BI gene locus with plasma lipid levels and body mass index in a white population. Arterioscler Thromb Vasc Biol 19(7):1734-1743

Barrett JC, Fry B, Maller J, Daly MJ (2005) Haploview: analysis and visualization of $\mathrm{LD}$ and haplotype maps. Bioinformatics 21(2):263-265

Bietrix F, Yan D, Nauze M, Rolland C, Bertrand-Michel J, Coméra C, Schaak S, Barbaras R, Groen AK, Perret B, Tercé F, Collet X (2006) Accelerated lipid absorption in mice overexpressing intestinal SR-BI. J Biol Chem 281(11):7214-7219

Boehnke M (1991) Allele frequency estimation from data on relatives. Am J Hum Genet 48(1):22-25

Brown MS, Goldstein JL (1986) A receptor-mediated pathway for cholesterol homeostasis. Science 232(4746):34-47

Cullen P, Funke H, Schulte H, Assmann G (1997) Lipoproteins and cardiovascular risk-from genetics to CHD prevention. J Atheroscler Thromb 4(2):51-58

Durstine JL, Grandjean PW, Davis PG, Ferguson MA, Alderson NL, DuBose KD (2001) Blood lipid and lipoprotein adaptations to exercise: a quantitative analysis. Sports Med 31(15):1033-1062

Feitosa MF, Rice T, Rankinen T, Province MA, Chagnon YC, Gagnon J, Leon AS, Skinner JS, Wilmore JH, Després JP, Bouchard C, Rao DC, Borecki IB (2005) Evidence of QTLs on chromosomes $13 \mathrm{q}$ and $14 \mathrm{q}$ for triglycerides before and after
20 weeks of exercise training: the HERITAGE Family Study. Atherosclerosis 182(2):349-360

Fisher RA (1934) Statistical methods for research workers. Oliver and Boyd, Edinburgh

Gordon DJ, Rifkind BM (1989) High-density lipoprotein-the clinical implications of recent studies. $N$ Engl $J$ Med 321(19):1311-1316

Hauser H, Dyer JH, Nandy A, Vega MA, Werder M, Bieliauskaite E, Weber FE, Compassi S, Gemperli A, Boffelli D, Wehrli E, Schulthess G, Phillips MC (1998) Identification of a receptor mediating absorption of dietary cholesterol in the intestine. Biochemistry 37(51):17843-17850

Kozarsky KF, Donahee MH, Rigotti A, Iqbal SN, Edelman ER, Krieger M (1997) Overexpression of the HDL receptor SR-BI alters plasma HDL and bile cholesterol levels. Nature 387(6631):414-417

Kuller LH, Simkin-Silverman LR, Wing RR, Meilahn EN, Ives DG (2001) Women's healthy lifestyle project: a randomized clinical trial: results at 54 months. Circulation 103(1):32-37

Lewontin RC (1964) The interaction of selection and linkage. II. optimum models. Genetics 50:757-782

Mero N, Van Tol A, Scheek LM, Van Gent T, Labeur C, Rosseneu M, Taskinen MR (1998) Decreased postprandial high density lipoprotein cholesterol and apolipoproteins A-I and $\mathrm{E}$ in normolipidemic smoking men: relations with lipid transfer proteins and LCAT activities. J Lipid Res 39(7):1493-1502

Ordovas JM (2001) Genetics, postprandial lipemia and obesity. Nutr Metab Cardiovasc Dis 11(2):118-133

Ordovas JM, Cupples LA, Corella D, Otvos JD, Osgood D, Martinez A, Lahoz C, Coltell O, Wilson PW, Schaefer EJ (2000) Association of cholesteryl ester transfer protein-TaqIB polymorphism with variations in lipoprotein subclasses and coronary heart disease risk: the Framingham study. Arterioscler Thromb Vasc Biol 20(5):1323-1329

Osgood D, Corella D, Demissie S, Cupples LA, Wilson PW, Meigs JB, Schaefer EJ, Coltell O, Ordovas JM (2003) Genetic variation at the scavenger receptor class B type I gene locus determines plasma lipoprotein concentrations and particle size and interacts with type 2 diabetes: the Framingham study. J Clin Endocrinol Metab 88(6):2869-2879

Otvos JD, Jeyarajah EJ, Bennett DW, Krauss RM (1992) Development of a proton nuclear magnetic resonance spectroscopic method for determining plasma lipoprotein concentrations and subspecies distributions from a single, rapid measurement. Clin Chem 38(9):1632-1638

Pankow JS, Province MA, Hunt SC, Arnett DK (2002) Regarding "Testing for population subdivision and association in four casecontrol studies". Am J Hum Genet 71(6):1478-1480

Pérez-Martínez P, Ordovás JM, López-Miranda J, Gómez P, Marín C, Moreno J, Fuentes F, Fernández de la Puebla RA, Pérez-Jiménez F (2003) Polymorphism exon 1 variant at the locus of the scavenger receptor class B type I gene: influence on plasma LDL cholesterol in healthy subjects during the consumption of diets with different fat contents. Am J Clin Nutr 77(4):809-813

Rigotti A, Trigatti BL, Penman M, Rayburn H, Herz J, Krieger M (1997) A targeted mutation in the murine gene encoding the high density lipoprotein (HDL) receptor scavenger receptor class B type I reveals its key role in HDL metabolism. Proc Natl Acad Sci USA 94(23):12610-12615

Stangl H, Cao G, Wyne KL, Hobbs HH (1998) Scavenger receptor, class B, type I-dependent stimulation of cholesterol esterification by high density lipoproteins, low density lipoproteins, and nonlipoprotein cholesterol. J Biol Chem 273(47):31002-31008

Suter PM, Gerritsen-Zehnder M, Häsler E, Gürtler M, Vetter W, Hänseler E (2001) Effect of alcohol on postprandial lipemia with and without preprandial exercise. J Am Coll Nutr 20(1):58-64 
Tsai MY, Yuan J, Hunninghake DB (1992) Effect of gemfibrozil on composition of lipoproteins and distribution of LDL subspecies. Atherosclerosis 95(1):35-42

Ueda Y, Royer L, Gong E, Zhang J, Cooper PN, Francone O, Rubin EM (1999) Lower plasma levels and accelerated clearance of high density lipoprotein (HDL) and non-HDL cholesterol in scavenger receptor class B type I transgenic mice. J Biol Chem 274(11):7165-7171

Uiterwaal CS, Grobbee DE, Witteman JC, van Stiphout WA, Krauss XH, Havekes LM, de Bruijn AM, van Tol A, Hofman A (1994) Postprandial triglyceride response in young adult men and familial risk for coronary atherosclerosis. Ann Intern Med 121(8):576-583

Wang N, Arai T, Ji Y, Rinninger F, Tall AR (1998) Liver-specific overexpression of scavenger receptor BI decreases levels of very low density lipoprotein ApoB, low density lipoprotein ApoB, and high density lipoprotein in transgenic mice. J Biol Chem 273(49):32920-32926

Yuan J, Tsai MY, Hunninghake DB (1994) Changes in composition and distribution of LDL subspecies in hypertriglyceridemic and hypercholesterolemic patients during gemfibrozil therapy. Atherosclerosis 110(1):1-11 